\title{
Telemedicine work station using computer communication network
}

\author{
K. Gugan ${ }^{1}$, R.G. Suresh Kumar ${ }^{2}$, S.Saravanan ${ }^{3}$ \\ AP, CSE Dept, Rajiv Gandhi college of Engg and Technology, Pondicherry university, Pudhucherry, India ${ }^{l}$ \\ AP(SL.G) , Rajiv Gandhi college of Engg and Technology, Pondicherry university, Pudhucherry, India ${ }^{2,3}$
}

\begin{abstract}
The increasing size of Biomedical Big data in Telemedicine Network server (TMNS) creates problem of over load and creates Transfer delay and CPU speed is decreased. This paper explains internet based Telemedicine workstation Setup Hybrid computing (Telemedicine computing, Cloud computing, Grid computing, Cluster computing) can be maintained complex Big Data storage of patient details like X-rays, Biomedical signals (ECG. EMG, Pulse, respiration)Blood pressure, Temperatures, Scanning reports to store in large capacity server in Hospital. Cloud computing Technology can be stored increasing large number of medical data and reduce $70 \%$ cost of server in Hospitals. Grid computing Technology can tally the over load balancing of Big data storage in CPU and increasing the CPU speed at Telemedicine work station. [1-7].
\end{abstract}

Keywords: Include 3G/4G Internet ,Grid computing , Cloud computing , Cluster computing

\section{INTRODUCTION}

Every day increasing the Patients Details of report, we Telemedicine work station (TWS) using computer need to store in various servers of Hospital. The single communication network through $3 \mathrm{G}$ Internet is now a node of server CPU speed is decreased by increasing proven technology that is capable of taking Health care overload storage of patient details and need to balance in reality by using Hybrid computing contains Grid, Cloud, servers. $2 \mathrm{G}$ internet speed is not enough for transmission Cluster and Telemedicine computing with information from sender to receiver and it will take max sec of time technology, medical devices, connectivity, communication delay. Now a days $3 \mathrm{G}$ internet speed is enough for technology and medical man ware. The implementation transmission from sender to receiver, the transmission and Telemedicine operational management can be simple delay is also very less. This paper explain in section I and prone to successful by good planned well [2]. The Telemedicine Hybrid computing consist of Grid, Cloud, Telemedicine workstation using Hybrid computing as Cluster computing for Big data store and overload shown in Figure 2

balancing and explain Transfer delay using various $3 \mathrm{G}$ internet speed. Section II explain the Architecture Model of Hybrid computing is shown in Figure 1.

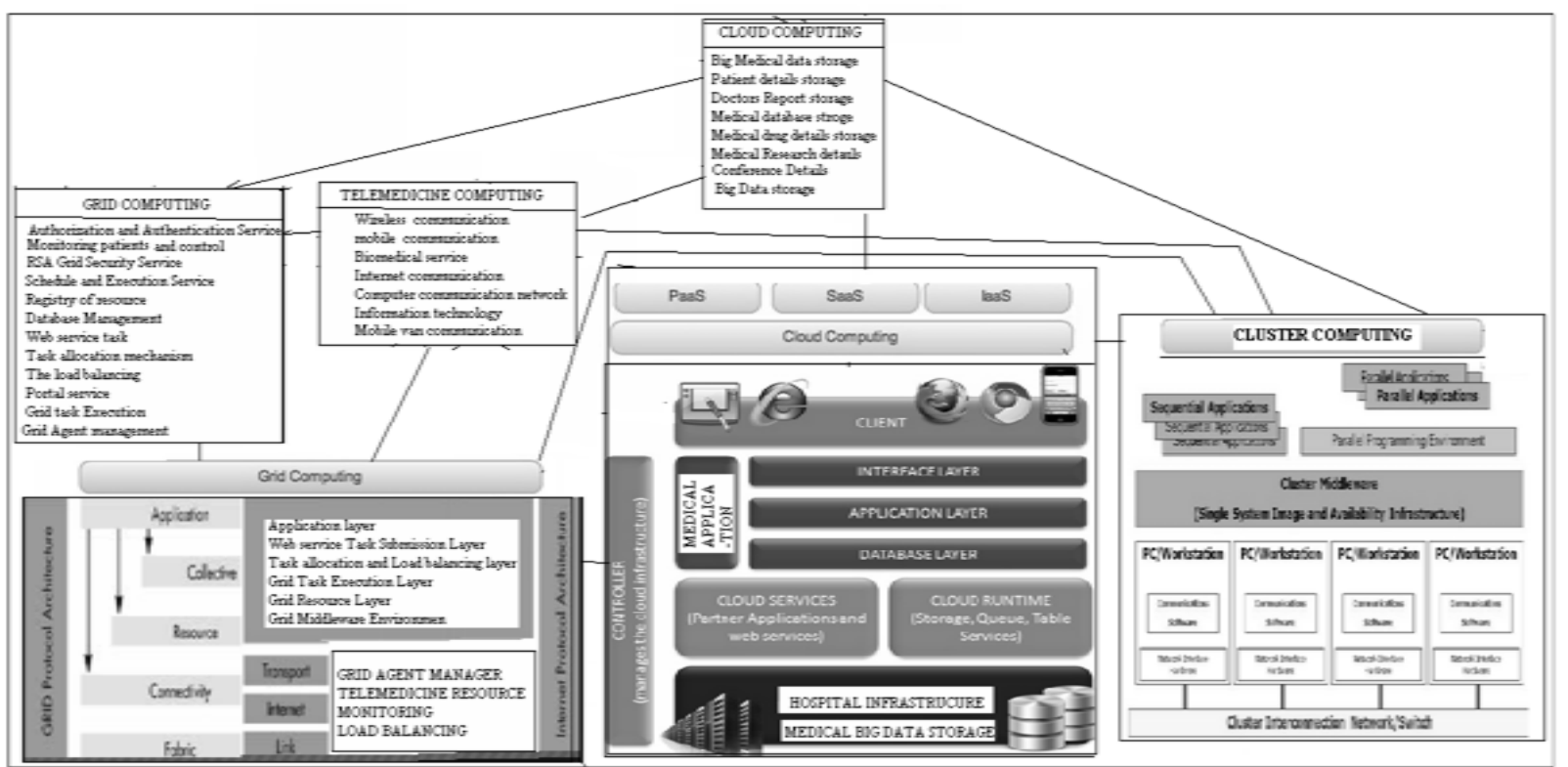

Fig1. Architecture Model of Hybrid computing 


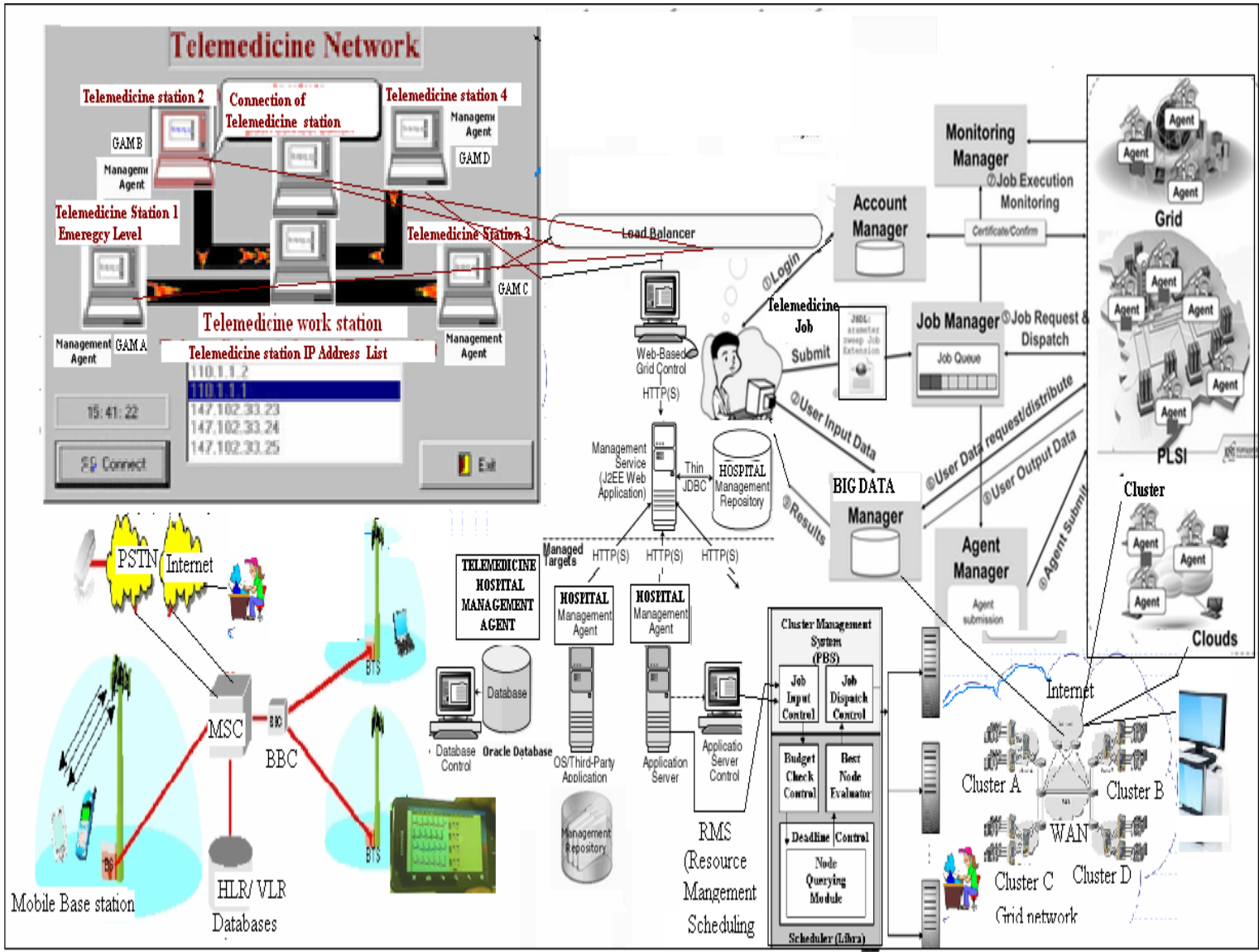

Fig. 2 Telemedicine work station using Hybrid computing

\section{A. Telemedicine computing}

The Telemedicine computing compute Transfer delay of dnodal $=$ dproc + dqueue + dtrans + dprop the Big data of patients details exchange from source to destination. If we transmit Bulk of Biomedical Image through $3 \mathrm{G}$ internet bandwidth is enough but it will take Transmission delay from $5 \mathrm{sec}$ to $33 \mathrm{sec}$ for image transmission. The propagation, Transmission, Queue delay are very small factor of delay but CPU processing delay creates big delay for big data transmission from source to destination in hospital.

Patient details of packet as it travels from Ambulance to Hospital, packet travels from Ambulance host or router to the subsequent node(host or router) along this path, the packet suffers from several different types of delays at each node along the path. The packet format of Biomedical data and image as shown in Figure 3. The most important of these delays are the nodal processing delay, queuing delay, transmission delay, and propagation delay.. The total nodal delay reduced by high speed $3 \mathrm{G}$ internet antenna in fixed in Ambulance bus.

\begin{tabular}{|c|c|c|c|}
\hline $\begin{array}{l}\text { Start of } \\
\text { message } \\
1 \text { byte }\end{array}$ & $\begin{array}{l}\text { Data } \\
\text { (Biomedical } \\
\text { data }+ \\
\text { Image) }\end{array}$ & $\begin{array}{l}\text { CRC check } \\
\text { CRC-Cyclic } \\
\text { Redundancy } \\
\text { Check) }\end{array}$ & $\begin{array}{c}\text { End of } \\
\text { message } \\
1 \text { byte }\end{array}$ \\
\hline
\end{tabular}

Fig.3 Packet format.

If we let dproc, dqueue, dtrans, and dprop denote the processing, queuing, transmission, and propagation delays, then the total nodal delay is given by

Example: Calculation of ECG nodal packet delay from Ambulance to hospital,

Assume 100 byte packet of ECG to be sent from ambulance to hospital distance $10 \mathrm{KM}$ away.

ECG signal Packet size $\mathrm{L}=100$ byte $=8 * 100$ $=800$ bits

3G Dialup Internet Modem of reliance Bandwidth $\mathrm{R}=$ 21.7 Mbps $=21.7 * 10^{6} \mathrm{Mbps}$

-dtrans $=\mathrm{L} / \mathrm{R}=0.000368 \mathrm{sec}$

$\mathrm{d}=$ Length of Physical link=Assume distance between ambulance to Hospital $=10 \mathrm{KM}=10000$ meters.

$\mathrm{S}=$ light speed $=2 * 10^{8} \mathrm{~m} / \mathrm{sec}$ to $3 * 10^{8} \mathrm{~m} / \mathrm{sec}$ $-d$ prop $=d / S=10000$ meters $/ 3 * 10^{8} \mathrm{~m} / \mathrm{sec}=$

$$
0.00003 \mathrm{sec} \text {. }
$$

-dproc $=0.15 \mathrm{sec}$ processing delay of normal Lap Top or personal computer. If we store large big data in CPU the processing delay increase $0.15 \mathrm{sec}$ to $10 \mathrm{sec}$, this maximum of CPU processing delay need to reduce by overload balance algorithm using Grid computing in next level)

-dqueue $=0.05 \mathrm{sec}($ The delay of queue of internet is busy or not free) 
$\mathrm{d}$ nodal $=$ dproc + dqueue + dtrans + dprop $=$

$$
\begin{aligned}
& 0.15 \mathrm{sec}+0.05 \mathrm{sec}+0.000368 \mathrm{sec}+0.00003 \mathrm{sec} \\
& =0.150398000 \mathrm{sec}=0.150 \mathrm{Sec}
\end{aligned}
$$

ECG total nodal delay packet of 100 byte $=0.15 \mathrm{sec}$ using 3G internet Reliance speed 21.7 Mbps. The above calculation is given the packet delay for Biomedical data and images are given Table 2. The CPU processing delay created by the large big data storage in server so create overload. The performance of CPU speed decreased by overload in single server in hospital. The over load of CPU speed performance increased by Grid computing algorithm support load balancing to implements to servers in Hospital and increased CPU speed. The Big data of patient details are stored in Cloud. So reduced CPU processing delay by avoid overload in CPU. The load balance with CPU speed as shown in Figure 4 .

\section{B. Grid and Cloud computing}

Telemedicine service and developed from virtualization, utility computing, IaaS ( Infrastructure as a Service ), PaaS (Platform as a Service), SaaS (Software as a Service ) to implemented in Telemedicine service using computer communication network and $3 \mathrm{G} / 4 \mathrm{G}$ internet.[3][4].

The Cloud computing hardware and platform level resources are provided as services on demand basis utilizes the high speed $3 G / 4 G$ Internet to deliver the computing, storage, software and services which are distributed in hospital.

\section{Telemedicine Job Parameters}

The following parameters are defined for Telemedicine System parameters

- Telemedicine Job Parameters: Telemedicine job of ID, Number of instruction per telemedicine Job, Job size of Telemedicine.

- Grid computing service (GCS):Telemedicine Task allocation mechanism allocate the TM node tasks by GCS and load balancing the servers of Hospitals. [3][4].

Processing Elements (PEs) Parameters: CPU speed, Index of Telemedicine work load jobs queued on a given PE and its CPU speed.

- Grid Agent Manager (GAM): The Load balancing layer proposed load balancing algorithm consists of GAM (Grid Agent Manager) which distributes the Telemedicine workstation

\section{TABLE I COMPARSION OF TRANSMISSION DELAY USING 3G} INTERNET

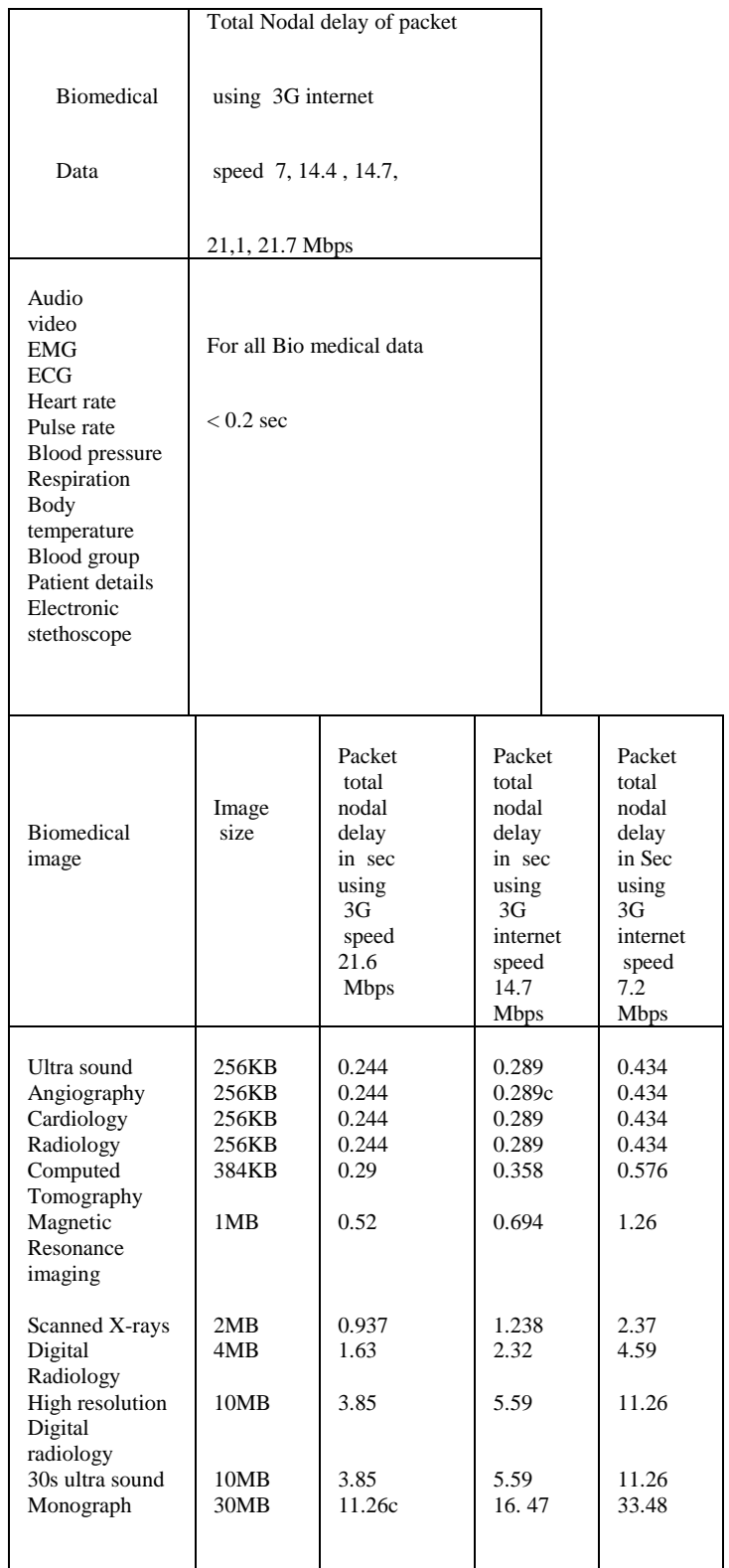

- Actual Processing Capacity (APC): Calculate the Number of Task per second in the Telemedicine workstation of Grid.

- Available Grid Processing capacity (AGPC): Calculate the available in Grid Telemedicine CPU Processing.

- Total Available Processing capacity (TAPC): Calculate the total of telemedicine task available process capacity in Telemedicine workstation of Grid.

- PE processing Capacity (PEPC): Number of Telemedicine Jobs per second in a processing elements is calculated using CPU speed and an average number of Instruction per Job. 
- Cluster Manager processing capacity (CMPC): Number of telemedicine jobs per second in the cluster in Telemedicine workstation can be calculated as the sum of PEPCs.

- Network Parameters: Band width of network. For example Reliance internet Modem bandwidth speed 21.6 Mbps and WAN internet bandwidth speed is $100 \mathrm{Mbps}$.

As Grid $\mathrm{G}$ of Telemedicine work station of hospital sections as a collection of $\mathrm{C}$ number of Cluster, Global scheduler (GS) who communicates through internet and computer communication network and the scheduling to Telemedicine task to the distributed Cluster of Telemedicine work station. Cluster consist of $\mathrm{N}$ number of Telemedicine processing Elements (PEs) and Local scheduler (LS) acts as a Cluster Manger (CM) and GS act as a Grid Agent Manager (GAM). There is a Telemedicine Job decides and arrival stream at LS, it dispatches the submitted telemedicine jobs to PE, LS handles inside hospital of Cluster communication and GS handles outside Hospitals of Cluster.

D. Grid Computing Load balancing algorithm

Mainly load balancing algorithm needs to increasing overload of un limited storage of patient Big-data in Hospital or Ambulance server and need to stores in Cloud through internet connection. Every GAM (Grid Agent Manager) sharing overloads by Grid computing service and minimize the congestion, waiting time, Communication delay. The Model of load balancing algorithm implements to Telemedicine workstation through 3G/4G Internet. [5][6][7].

Example: If Grid Telemedicine work station of Grid Manager consists of GAMs A, B, C, D... are shares TM Tasks and overloads by Grid computing.

Actual processing capacity (APC) of Grid manger at Telemedicine workstation of Grid system $(\mathrm{APC})=\mathrm{PC} * \mathrm{~L}$

\section{- if $P C=500$ Tasks $/ \mathrm{sec} L=30 \% \quad A P C=500 * 30 / 100=150$ \\ Tasks/sec

- If implements the Grid Computing method ,the Telemedicine work station of Grid Threshold is assume $80 \%$ then available Grid processing capacity (AVGPC)= GPC-APC =

$$
=500 * 80 / 100-150=250 \text { Tasks } / \text { sec. }(2)
$$

- If Telemedicine Branch GAM A receveived 200 Tasks of TM nodes and it has three receptors $r 1, r 2$, and $r 5$ with AVGPC of 250,300 and 140 tasks/sec respectively then,

- TAPC(Total available processing capacity)

$$
=\sum \underset{\mathrm{r}=1}{\mathrm{AVGPC}(\mathrm{r})}
$$

$=250+300+140=690$ Tasks $/ \mathrm{sec}$

Shares $\mathrm{r}(1)=(250 / 690) * 200=727$ Tasks

Shares $\mathrm{r}(3)=(300 / 690) * 200=87$ Tasks

Shares $\mathrm{r}(5)=(140 / 690) * 200=41$ Tasks

Copyright to IJARCCE
- Use If GAM A has $\mathrm{N}=100$ unprocess of telemedicine work station nodes tasks the following GAM load will distribute as follows. The GAM status as shown in Table 2 .

TABLE II GAM STATUS

\begin{tabular}{|c|c|c|c|c|}
\hline \multirow{2}{*}{ TM Grid } & \multicolumn{4}{|c|}{ Grid TM Network of Grid Agent manager(GAM) of id } \\
\cline { 2 - 5 } Manager in & GAM A & GAMB & GAB C & GAMD \\
$\begin{array}{r}\text { Grid TM } \\
\text { Network }\end{array}$ & & & & \\
\hline Status & S & R & R & R \\
\hline TAPC & 1000 & 700 & 550 & 600 \\
\hline
\end{tabular}

Sender(s) in Telemedicine station: The Sender may be Ambulance or Hospital server of small TAPC (Telemedicine Actual Processing capacity) and the pool of TM nodes workers are over loaded need to transfer of load to GAMs.

Receivers in Telemedicine station: Available TAPC is high, ready to receive new tasks and thus increase the throughput

Neutral $(\mathrm{N})$ : Pool under normal load of TM nodes in workstation

\section{E. Telemedicine work station of Grid Task}

- GAM B can offer up to 700 Telemedicine tasks/sec but since we can transfer a maximum of 200 Telemedicine tasks /sec over the link connecting GAM A and GAM B then GAM B can only supply 200 Telemedicine Tasks/sec

- GAM C can offer up to 550 Telemedicine tasks/sec and since the link can offer that amount the GAM C can supply the 550 tasks/sec

- GAM D offer up to 600 Telemedicine tasks /sec and since the link can offer that amount the GAM D can supply the 600 tasks /sec

- Telemedicine work station of Grid Total available processing Capacity (GTAPC)

$=200+550+600=1350 \mathrm{Tasks} / \mathrm{sec}$.

- GAM B $=(200 / 1350) * 1000=149$ Telemedicine Tasks GCS (Grid computing service) allocates the task to suitable TM resources then the task needs to be executed.

- Calculated average TM task size , average task execution time and updated at the GAM levels. Calculated Determine the computing capacity (number of task per unit of time) at a given computing telemedicine center Share GAM $\mathrm{C}=(550 / 13508) * 1000=407$ Telemedicine Tasks.

Share GAM D $=(600 / 1350) * 1000=444$ Telemedicine Tasks

F. Telemedicine work Station of Grid Resource Layer

- GAM are building blocks of TM resource monitoring layer and computing different Hospitals computing sites of networks technologies and different operating system. 
- GAM in the TM Grid system exchanges Big data to achieve load balancing operation of TM work station of center.

- Maintaining the Computers in workstations, Grid and Clusters of computers in Telemedicine network through Internet

- Maintaining the Big Data resources, Grid configurations and log history of the TM events.

- Maintaining Databases of the Telemedicine server Big data related to generation, transmission and load demand.

\section{G. Cluster computing load balancing}

Define the Telemedicine PE share of each PE in a pool the following by

\section{PE share ${ }_{i=}$ NC. PEPC $/$ CMPC}

Where PE share $i_{I}=$ Processing Element of Telemedicine Share of $\mathrm{i}$.

$\mathrm{C}=$ Number of cluster

$\mathrm{N}=$ Number of telemedicine Jobs.

$\mathrm{PEPC}=$ Processing Elements of Processing Capacity.

$\mathrm{CMPC}=$ Cluster Manager Processing Capacity. Local Scheduler (LS) acts as to check its Telemedicine resources are overloaded by capacity work load index of PE and its PE share.

Consider the distribution of Telemedicine Job J submitted in Grid node $\mathrm{G}$ from Cluster $\mathrm{C}$, The Completion Time of telemedicine $\mathrm{Job} \mathrm{J}$ is following formula.

Completion time $(\mathrm{J})=$

Runtime(J).Runqueuelenth $(\mathrm{C}) / \operatorname{Speed}(\mathrm{C})+$

Jobsize(J)/ Link speed $(\mathrm{G}, \mathrm{C})$

Run time $(\mathrm{J})=$ Execution time of Telemedicine Jobs $\mathbf{J}$ runs on a computer server.

Job size $(\mathbf{J})=$ Total Telemedicine Job size

Link Speed $(\mathrm{G}, \mathrm{C})=$ Speed of Communication link between $\operatorname{Grid}(\mathrm{G})$ and Cluster $(\mathrm{C})$ in Mbps in Telemedicine Network.

Run queue length $(\mathrm{C})=$ Current run queue length of Cluster (C).

Speed $(\mathrm{C})=$ Speed of Cluster $\mathrm{C}$.

Mean Response Time $(\mathrm{RT})=$ $\mathrm{N}$

$=1 / \mathrm{N}\left(\sum_{\mathrm{j}=1} \mathrm{r}_{\mathrm{j}}\right)$

where $\mathrm{j}=$ the Response time of telemedicine Job $\mathrm{j}$

spent in the Resource of queue.

Slow down is defined in Telemedicine Network as follows

$\mathrm{S}_{\mathrm{j}}=\mathrm{r}_{\mathrm{j}} / \mathrm{e}_{\mathrm{j}}$

where

$\mathrm{r}_{\mathrm{j}=}$ Response time of Job $\mathrm{j}$.

$\mathrm{e}_{\mathrm{j}=}$ Execution time of $\mathrm{j}$
The average slow down SLD is defined

$$
\mathrm{N}
$$

$\mathrm{SLD}=1 / \mathrm{N}\left(\sum_{\mathrm{i}=1} \mathrm{~S}_{\mathrm{j}}\right)$

$\mathrm{N}=$ The Total number of processing Jobs.

The Load information Traffic (LIT) is defined as follows

e

$\mathrm{LIT}=\sum_{1}(\mathrm{C}, \mathrm{W})$

Where $\mathrm{C}$ is the number of Cluster.

$\mathrm{W}$ is the message weight from each Cluster.

$\mathrm{e}$ is the number of load information exchange events occurred until the end of telemedicine processing.

\section{H. Grid Middleware environment}

This layer provides a link between the Hospital Grid Telemedicine Network services and Grid Telemedicine resources. It provides the access and information about the Grid resources to the grid services in layer. This layer consists of RMI and JINI technology, which provides standard distributed services. This layer consists of Globus Toolkit software 4.0, which is standard OGSA platform and provides standard services [5][6][7].

\section{Page Style Performance of big data and load balancing IN GRID COMPUTING IN HOSPITAL}

The various section of reports like as X-rays, scanner image, angiography, cardiology, radiology, computed Tomography, Magnetic resonance image, Digital radiology, Monograph, Blood bank, Lab data, Result Reporting, Medical Dispensing, surgery scheduling and management, Supplies management, telephone console, Material management, patient scheduling, compliance checking, Patient monitoring, Lab information system, Disease Management, Medical transcription, ER patient Tracking and ER Management, compliance checking, Monitoring heart patients, Monitoring Patients in ambulance are to be Stored in hospital servers. Figure 3 presents a common healthcare data integration architecture for Big data servers and load balancing by load balancer. This environment includes various section hospitals of servers by point-to-point communication of interfaces through internet to a Big data interface Grid server as shown in Figure 2. The Load Balancing of different section of hospital Servers CPU status as shown in Figure 4 and resource status as in Figure 5.

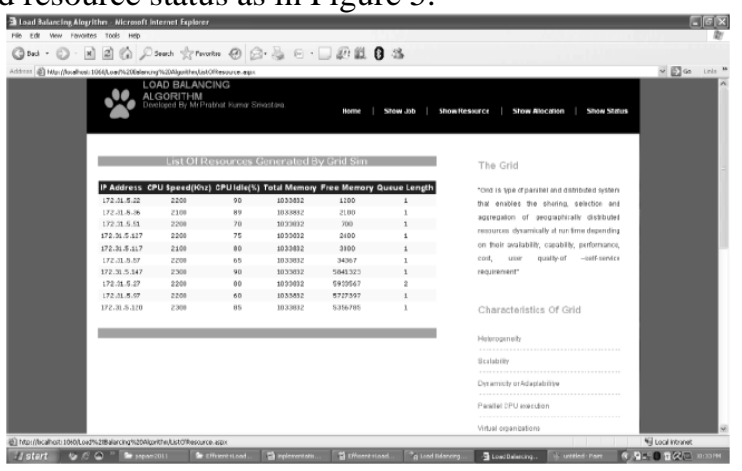

Fig 4 Load balancing of CPU status 
In Figure 4 shows the performance of CPU speed 3. increased by load balance by Grid computing algorithm

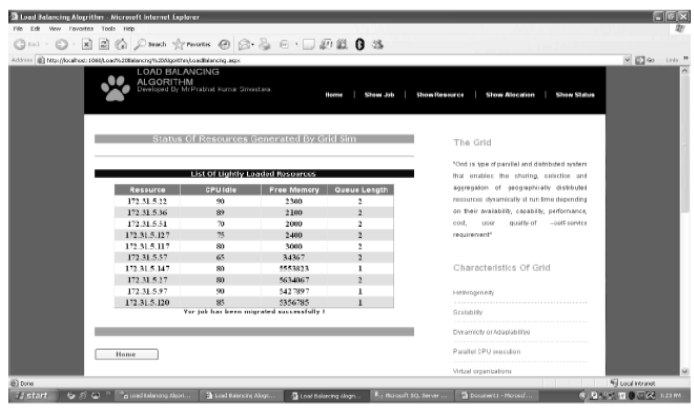

Fig 5 Telemedicine Hospital Resource Status.

In Figure 5 shows the various resource node available of Hospitals.

\section{CONCLUSION}

The Telemedicine Base Station using computer communication network and Cloud and Grid computing Support following:

- Reduced system cost: The big data of server cost reduced $70 \%$ of by Cloud storage.

- Store Large Big Data of Medical data: In day by day increasing the patients Record and Medical data and image of Big data can be stored in servers and load balance by Grid computing then store to cloud. So We can store the last 35 years of patients details can be stored in cloud and retrieve the patients details at any time and anywhere from Cloud with security.

- Maintenances and communication Cost of Telemedicine workstation using 3G/4G Internet: The Maintenance cost of TM workstation through 3G/4G internet is very low comparably VSAT based TM network. - Reduced Transmission Delay: The processing CPU speed increased by load balancing algorithm. The transmission delay reduced by $3 \mathrm{G}$ internet Modem with Antenna. In practically 0.15 to $2 \mathrm{sec}$ transmission delay of ECG signal using 3G internet of 21.7 Mbps [8]

\section{ACKNOWLEDGMENT}

I would like to S.SARAVANAN, AP(SL>G), CSE Dept., R.G Suresh Kumar, Head of the Computer science department, Rajiv Gandhi college of Engineering and Technology, Puducherry, India. They Guide to Telemedicine base station of center.

\section{REFERENCES}

1. Perumal.B Ramalingam.H.M, Department of Electronics and Communication Engineering, Kalasalingam University, Pallikonda Rajasekaran .M , Department of Instrumentation and Control Engineering, Kalasalingam University, WSN INTEGRATED CLOUD FOR AUTOMATED TELEMEDICINE (ATM) BASED e-HEALTHCARE APPLICATIONS, 2012 4th International Conference on Bioinformatics and Biomedical Technology IPCBEE vol.29 (2012) @ (2012) IACSIT Press, Singapore

2. NEHA BISHNOI \& ANUPMA SEHRAWAT,Department of Computer Science, Amity University, Gurgaon, Haryana, India, CLOUD COMPUTING ARCHITECTURE TO AID TELEMEDICINE, International Journal of Computer Science Engineering and Information Technology Research (IJCSEITR) ,ISSN 2249-6831,Vol. 3, Issue 4, Oct 2013, 13-18, @ TJPRC Pvt
Mr. Amitav Mahapatra,Lecturer, College of Engineering and Technology, Bhubaneswar, Mr. Manoranjan Dash,Asst.Professor, Institute of Business and Computer Studies, SOA University, Bhubaneswar ,Design and Implementation of a Cloud based TeleDermatology System, International Journal of Engineering Research \& Technology (IJERT), Vol. 2 Issue 2, February2013,ISSN: 2278-0181

4. Carmelo Pino and Roberto Di Salvo,Department of Electrical, Electronics and Computer Engineering (DIEEI) University of Catania, V.le Andrea Doria, 6 - 95125 (Italy),\{cpino \& disalvo\}@dieei.unict.it, A Survey of Cloud Computing Architecture and Applications in Health, 2nd International Conference on Computer Science and Electronics Engineering (ICCSEE 2013).

5. Sandip Kumar Goyal and Manpreet Singh. Department of Computer Science \& Engineering, M. M. Engg. College,M. M. University, Mullana, Ambala, Haryana 133203, India, Enhanced Genetic Algorithm Based Load Balancing in Grid, IJCSI International Journal of Computer Science Issues, Vol. 9, Issue 3, No 2, May 2012,ISSN (Online): 1694-0814.

6. Rafiqul Z. Khan ,Department of Computer Science, Aligarh Muslim University, Aligarh, India ,Email: rzk32@yahoo.co.in ,Md F. Ali Department of Computer Science, Aligarh Mslim University, Aligarh, India ,Email: firojali.mca@gmail.com, An Efficient Diffusion Load Balancing Algorithm in Distributed System, I.J. Information Technology and Computer Science, 2014, 08, 65-71 Published Online July 2014 in MECS (http://www.mecs-press.org/) DOI: $10.5815 /$ ijitcs.2014.08.09

7. Neeraj Nehra, R.B.Patel, V.K.Bhat, Distributed Parallel Resource Co-Allocation with Load Balancing in Grid Computing, IJCSNS International Journal of Computer Science and Network Security, VOL.7 No.1, January 2007.

8. S.Saravanan, Mr.Pillutla Harikrishna, Dr. J Vaideeswaran, "Big data exchange between Ambulance Bus to Hospital network through internet in Telemedicine using computer communication network and 3G Mobile Antenna" IEEE, 2015 International conference on Computer communication and informatics (ICCCI2015), Jan 8-10 , coimbatore, INDIA.

\section{BIOGRAPHIES}

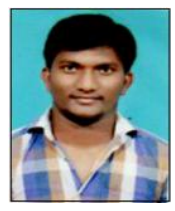

Mr.K. Gugan has received B.Tech (I.T), M..Tech (CSE) in Pondicherry University, Puducheery $\mathrm{He}$ has interest in areas of Image processing, Grid computing, cloud computing, Data Mining. Telemedicine network. He has done Data Mining Project in UG level and Image Processing Project in PG level.

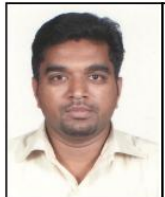

Mr. Suresh Kumar.RG MCA, MTech obtained his Master of Technology from Faculty of Information Technology, Allahabad agricultural University in 2008. Currently, he is Assistant Professor (SL.G) at Department of Computer Sciences, Rajiv Gandhi College of Engineering And Technology. He is a Sun Certified Professional and His main research areas include Mobile computing, Cloud computing and Wireless networks

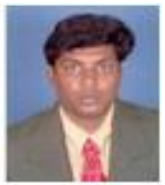

Mr. S. Saravanan, Asst. Professor (selection Grade) in computer science Department in Rajiv Gandhi college of Engineering and technology, puducherry, India. He has completed B.E( Electronics and communication) in 1998, M.S (Information technology) in 2003, M.E ( computer science Engineering) in 2008, MBA(Education management)in 2010, M.Tech ( Communication System) in 2012,.Ph.D pursuing from 2009. He has teaching experience from 1998 in various Department of Electronics and communication, Biomedical Engineering and Computer science Engineering. 